\title{
The separable Jung constant in Banach spaces
}

\author{
Jesús M. F. Castillo and Pier Luigi Papini
}

\begin{abstract}
This paper contains a study of the separable version $J_{s}(\cdot)$ of the classical Jung constant. We first establish, following Davis [15, that a Banach space $X$ is 1-separably injective if and only if $J_{s}(X)=1$. This characterization is then used for the understanding of new 1-separably injective spaces. The last section establishes the inequality $\frac{1}{2} K(Y) J_{s}(X) \leq e_{1}^{s}(Y, X)$ connecting the separable Jung constant, Kottman's constant and the separable-one-point extension constant for Lipschitz maps, which is then used to derive an improved version of Kalton's inequality $K\left(X, c_{0}\right) \leq e\left(X, c_{0}\right)$ and a new characterization of 1-separable injectivity.
\end{abstract}

\section{The Jung constants}

Given a bounded subset $A \subset X$ we define the diameter of $A$ as $\delta(A)=\sup \{\| a-$ $b \|: a, b \in A\}$, while the radius of $A$ is defined by $r(A)=\inf _{b \in X} \sup _{a \in A}\|a-b\|$. The Jung constant 24] of $X$ is defined as

$$
J(X)=\sup \frac{2 r(A)}{\delta(A)}
$$

where the supremum is taken over all closed bounded sets $A$ with $\delta(A)>0$. It was shown by Davis in [15] and by Franchetti in [19] that a Banach space with Jung constant 1 is 1-injective. It follows then from the work of Lindenstrauss [27] that a Banach space is 1-injective if and only if $J(X)=1$. Recall that a Banach space $X$ is $\lambda$-injective if for every Banach space $F$ and every subspace $E$ of $F$ every operator $t: E \rightarrow X$ has an extension $T: F \rightarrow X$ with $\|T\| \leq \lambda\|t\|$.

Two important variations of this notion are: $\lambda$-separable injectivity, when the property above holds when $F$ is separable; and universal $\lambda$-separable injectivity [5, 8], when the preceding property holds when $E$ is separable. Other cardinal variations

2010 Mathematics Subject Classification. 46B04, 46B20, 46B26, 46M18 .

Key words and phrases. 1-separable injectivity; Jung constant; Kottman constant.

The research of the first author was supported in part by Project IB16056 de la Junta de Extremadura and MINCIN Project MTM2016-76958-C2.

The authors thank the referee for his/her splendid job and many poignant comments that helped enormously to improve the quality of the paper. 
of this notion were studied in [7] and [8]. Accordingly, given an uncountable cardinal $\aleph$, a Banach space $X$ is said to be $(\lambda, \aleph)$-injective if the preceding condition holds for Banach spaces $F$ with density character less than $\aleph$. As it is remarked in $[\mathbf{8}$, Def. 5.1], "the resulting name for separable injectivity turns out to be $\aleph_{1}$-injectivity, not $\aleph_{0}$-injectivity, as one would have expected. Nevertheless, we have followed the uses of set theory where properties labeled by a cardinal always indicate that something happens for sets whose cardinality is strictly less than that cardinal".

Turning back to the connections between injectivity properties and the Jung constant, recall that a Banach space is 1-injective if and only if every family of mutually intersecting balls has nonempty intersection and observe that 1-injective spaces are 1-complemented in any larger superspace; in particular, in larger one codimensional superspaces ( $F$ is a one codimensional superspace of $E$ if $\operatorname{dim} F / E=$ 1 ). This is the core of the proof that 1-injectivity can be characterized by properties of intersection of balls of equal radius. Namely, a Banach space is 1-injective if and only if every family of mutually intersecting balls of radius one admits nonempty intersection; i.e., $J(X)=1$. Such is the content of the proof of Franchetti [19]: indeed, it is based on the estimate $1 \leq J(X) \leq \lambda_{1}(X) \leq g(J(X))$ where $g:[1,2] \rightarrow$ $[1,2]$ is a certain function such that $g(1)=1$.

What occurs under cardinal restrictions? For instance, are the properties every countable family of mutually intersecting balls has nonempty intersection and every family of mutually intersecting balls of radius one has nonempty intersection equivalent? The former of those properties corresponds [8] to 1-separable injectivity. To treat the latter we introduce the separable Jung constant $J_{s}(\cdot)$ defined as $J(\cdot)$ but considering only separable bounded sets (there is an implicit approach to this notion in $[10]$ ).

Definition 1. Given an uncountable cardinal $\aleph$ we define the Jung constant

$$
J_{\aleph}(X)=\sup \frac{2 r(A)}{\delta(A)}
$$

where the supremum is taken over all closed bounded sets $A$ with $\delta(A)>0$ and density character strictly less than $\aleph$.

Thus, $J_{s}(X)=J_{\aleph_{1}}(X)$; and the question is then whether $J_{s}(X)=1$ characterizes 1-separable injectivity. The straight way to derive this result from the injectivity result cannot work because, suprising as it may seems, 1-separably injective spaces are not necessarily 1-complemented in one-codimensional superspaces (see the proof below) and this is what makes difficult to find a way to adapt Franchetti's proof for separable injectivity. Davis proof [15] again relies on the fact of proving that spaces $X$ with $J(X)=1$ are 1 complemented in any superspace $F$ so that $\operatorname{dim} F / X=1$ and therefore it cannot work under cardinal restrictions. To make sound all these assertions, we need a couple of elements of homological Banach space theory. The 
first one is the notion of exact sequence $0 \rightarrow Y \rightarrow X \rightarrow Z \rightarrow 0$, which is a diagram formed by Banach spaces and linear continuous operators with the property that the kernel of each arrow coincides with the image of the preceding. Thanks to the open mapping theorem this exactly means that $Y$ is (isomorphic to) a subspace of $X$ and $Z$ is (isomorphic to) the corresponding quotient $X / Y$. An exact sequence is said to split if the subspace $Y$ is complemented in $X$; i.e., there is a linear continuous projection $P: X \rightarrow Y$. The second thing is the push-out construction (see complete details in [8]), which operatively defined means that whenever one has an exact sequence $0 \rightarrow Y \rightarrow X \rightarrow Z \rightarrow 0$ and an operator $\tau: Y \rightarrow Y^{\prime}$ there is a commutative diagram

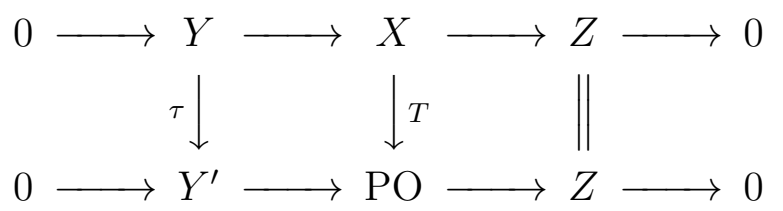

The push-out space PO is the quotient $\left(Y^{\prime} \oplus_{1} X\right) / \Delta(Y)$ where $\Delta: Y \rightarrow Y^{\prime} \oplus_{1} X$ is the isomorphic embedding $\Delta(y)=(\tau(y),-y)$. What is required to know to follow the arguments in this paper is:

(1) The lower sequence in diagram (1) is exact.

(2) Although, as we said before, the operator $Y \rightarrow X$ in an exact sequence as above only has to be an isomorphic embedding, in the exact sequences we will consider it is an isomeric embedding. When the inclusion $Y \rightarrow X$ (resp $\tau$ ) is an into isometry, the same occurs to the inclusion $Y^{\prime} \rightarrow \mathrm{PO}$ (resp. to $T)$.

(3) $T$ is always a contractive operator.

(4) The lower sequence in a push-out diagram as above splits if and only if $\tau$ admits a linear continuous extension $\widehat{\tau}: X \rightarrow Y^{\prime}$.

All these facts can be encountered with proofs in [8]. We prove the announced result. We are sure it is well known; but since we could not find an explicit reference, we include its proof for the sake of completeness.

LEMma 1. A Banach space $X$ is 1-injective if and only if it is 1-complemented in every one codimensional superspace.

Proof. One implication, as it has already been mentioned, is clear. Assume that $X$ is 1-complemented in every one codimensional superspace and let us show that every operator $\tau: E \rightarrow X$ admits an equal norm extension to any one codimensional superspace $F$ of $E$. Form the push-out diagram

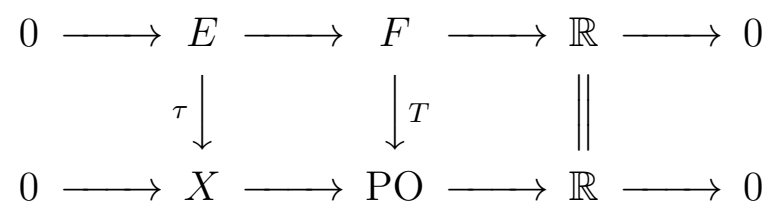


which clearly means that $X$ is of codimension 1 in PO. Let $P: \mathrm{PO} \rightarrow X$ be a norm one projection. The composition PT yields the equal norm extension of $\tau$.

Since, on the other hand, exist 1-separably injective spaces that are not 1-injective [5, 8], one gets that 1-separably injective are not necessarily 1complemented in one codimensional superspaces. Let us show that Davis proof can be nevertheless modified to cover the separable (as well as other cardinal restrictions) case. Precisely:

THEOREM 1. Let $\aleph$ be an infinite cardinal. A Banach space $X$ is $(1, \aleph)$-injective if and only if $J_{\aleph}(X)=1$. In particular, $X$ is 1-separably injective if and only if $J_{s}(X)=1$.

PROOF. We will make the proof for $\aleph=\aleph_{1}$ that corresponds to separable injectivity and indicate the modifications to be made for other cardinals. What we will actually show is that $J_{s}(X)=1$ implies that every norm one operator $\tau: E \rightarrow X$ with $E$ separable (in the general case will be $E$ having density character $<\aleph$ ) admits, for every $\varepsilon>0$, an extension $\tau_{\varepsilon}: F \rightarrow X$ with $\left\|\tau_{\varepsilon}\right\| \leq 1+\varepsilon$ whenever $F$ is a superspace of $E$ with $\operatorname{dim} F / E=1$. So we set $\mathbb{R}=F / E$ and denote by $f: F \rightarrow \mathbb{R}$ the quotient map. Form the commutative diagram

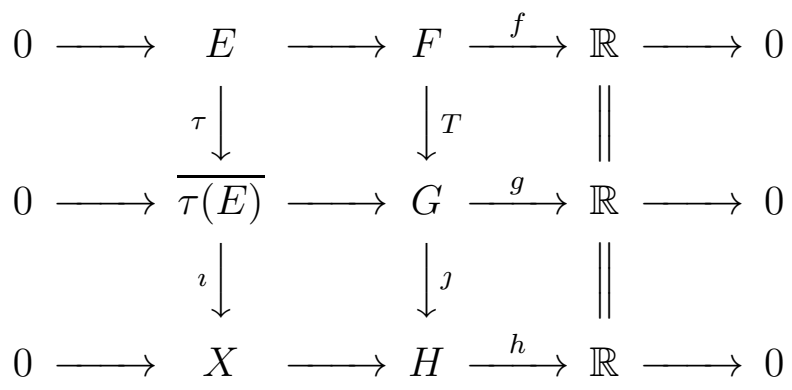

in which $G$ and $H$ are the corresponding push-out spaces and we have labeled $g, h$ the respective quotient maps. The operator $T$ has norm one and $\jmath$ is an isometric embedding, which clearly shows that it is enough to obtain an extension $\imath_{\varepsilon}: G \rightarrow X$ of $\imath$ with norm at most $1+\varepsilon$. To this purpose, observe that for each $0<\varepsilon<1$ the metric space $h^{-1}(\varepsilon)$ is isometric to $X$. Thus, $B(0,1) \cap h^{-1}(\varepsilon) \cap \jmath(G)$ is a (separable) set of diameter at most 2 of $X$ must have radius 1 ; which means that there exists $z_{\varepsilon}$ in $h^{-1}(\varepsilon)$ so that $B(0,1) \cap h^{-1}(\varepsilon) \cap \jmath(G) \subset B\left(z_{\varepsilon}, 1\right)$. We define an extension $\imath_{\varepsilon}: G \rightarrow X$ of the canonical inclusion $\imath$ as

$$
\imath_{\varepsilon}(x)=\jmath(x)-\frac{g(x)}{\varepsilon} z_{\varepsilon} .
$$

Claim 1. If $\|x\| \leq 1$ and $g(x)=\varepsilon$ then $\left\|\imath_{\varepsilon}(x)\right\| \leq 1$. 
Proof of the Claim. Pick $\|x\| \leq 1$. If $g(x)=\varepsilon$ then $x \in G \cap B(0,1) \cap g^{-1}(\varepsilon)$ and thus $\jmath x \in \jmath(G) \cap B(0,1) \cap h^{-1}(\varepsilon)$ so that $\left\|\imath_{\varepsilon}(x)\right\|=\left\|\jmath(x)-z_{\varepsilon}\right\| \leq 1$ by the choice of $z_{\varepsilon}$.

Now, if $\beta>\varepsilon$ then define the map $\phi_{\beta}: h^{-1}(\varepsilon) \rightarrow h^{-1}(\beta)$ given by

$$
\phi_{\beta}(y)=y+\frac{\beta-\varepsilon}{\varepsilon} z_{\varepsilon} \text {. }
$$

Let us check that if $\|x\|=1$ then $\left\|\phi_{\beta}(\jmath(x))\right\| \geq 1$. When $x \in g^{-1}(\varepsilon)$ one has $\imath_{\varepsilon}(x)=\jmath(x)-z_{\varepsilon}$ and thus it turns out that

$$
\begin{aligned}
\jmath(x) & =\frac{\beta-\varepsilon}{\beta}\left(\jmath(x)-z_{\varepsilon}\right)+\left(1-\frac{\beta-\varepsilon}{\beta}\right)\left(\jmath(x)+\frac{\beta-\varepsilon}{\varepsilon} z_{\varepsilon}\right) \\
& =\frac{\beta-\varepsilon}{\beta} \imath_{\varepsilon}(x)+\left(1-\frac{\beta-\varepsilon}{\beta}\right) \phi_{\beta}(\jmath(x)) .
\end{aligned}
$$

Therefore, if $\|x\|=1$ and $g(x)=\varepsilon$, since $\left\|\imath_{\varepsilon}(x)\right\| \leq 1=\|\jmath(x)\|$, one then has $\left\|\phi_{\beta} \jmath(x)\right\| \geq 1$ as we claimed.

Claim 2. $\jmath\left(B(0,1) \cap g^{-1}(\beta)\right) \subset \phi_{\beta} \jmath\left(B(0,1) \cap g^{-1}(\varepsilon)\right)$.

Proof of the Claim. To simplify the exposition of this part let us call $B_{\varepsilon}=$ $B(0,1) \cap g^{-1}(\varepsilon)$. The statement to prove is then $\jmath\left(B_{\beta}\right) \subset \phi_{\beta} \jmath\left(B_{\varepsilon}\right)$. Consider the convex set

$$
\mathcal{C}=\bigcup_{z \in B_{\varepsilon}}\left[\imath_{\varepsilon}(z), \phi_{1} \jmath(z)\right]=\jmath\left(B_{\varepsilon}\right)+\left[-z_{\varepsilon}, \frac{1-\varepsilon}{\varepsilon} z_{\varepsilon}\right]
$$

Moreover

$$
\phi_{\beta} J\left(B_{\varepsilon}\right)=\mathcal{C} \cap h^{-1}(\beta)
$$

since clearly $\phi_{\beta} \jmath\left(B_{\varepsilon}\right) \subset h^{-1}(\beta)$ and also picking $z \in B_{\varepsilon}$

$$
\phi_{\beta} \jmath(z)=(1-\beta)\left(\jmath z-\frac{g(z)}{\varepsilon} z_{\varepsilon}\right)+\beta\left(\jmath z+\frac{1-\varepsilon}{\varepsilon} z_{\varepsilon}\right) \in\left[\imath_{\varepsilon}(z), \phi_{1} \jmath(z)\right] \subset \mathcal{C} .
$$

This proves the inclusion $\subset$. Now we are going to prove the claim itself. Pick a point $\jmath(x) \in \jmath\left(B_{\beta}\right)$ and assume it is not in $\phi_{\beta} \jmath\left(B_{\varepsilon}\right)=\mathcal{C} \cap \jmath g^{-1}(\beta)$. This means that if one picks a point $y \in B_{\varepsilon}$ with $\|y\|<1$ - which must be in the interior of $\mathcal{C}$ - then the segment $[\jmath(y), \jmath(x)]$ must intersect the boundary of $\mathcal{C}$, which is the union of the three sets

$$
\begin{aligned}
& \mathcal{C}_{1}=\left\{\imath_{\varepsilon}(z): z \in B_{\varepsilon}\right\} \\
& \mathcal{C}_{2}=\left\{\phi_{1} \jmath(z): z \in B_{\varepsilon}\right\} \\
& \mathcal{C}_{3}=\bigcup_{\|z\|=1 ; z \in B_{\varepsilon}}\left[\imath_{\varepsilon}(z), \phi_{1} \jmath(z)\right]
\end{aligned}
$$


Since $h \jmath(y)=g(y)=\varepsilon$ and $h \jmath(x)=g(x)=\beta$ all points $u \neq \jmath(y)$ in $[\jmath(y), \jmath(x)]$ have $\varepsilon<h(u)<\beta$; so $[\jmath(y), \jmath(x)] \cap \mathcal{C}_{1}$ is impossible since $h \imath_{\varepsilon}(z)=0$ for every $z \in \mathcal{C}_{1}$. The intersection $[\jmath(y), \jmath(x)] \cap \mathcal{C}_{2}$ must also be empty since $h \phi_{1} \jmath(z)=g \phi_{1}(z)=\beta$ for every $z \in \mathcal{C}_{2}$. The only remaining possibility is the existence of some $u \in[\jmath(y), \jmath(x)] \cap \mathcal{C}_{3}$. And this is impossible because it forces $u$ to have $\|u\| \geq 1$ : indeed, recall that, as we showed above, $\jmath(z)$ is the norm one point in the interval $\left[\imath_{\varepsilon}(z), \phi_{1} \jmath(z)\right]$, and $h \jmath(z)=\varepsilon$. Assume $u \in(\jmath(y), \jmath(x)) \cap \mathcal{C}_{3}$. Since $\|\jmath(y)\| \leq 1$ and $\|\jmath(x)\| \leq 1$, necessarily $\|u\|<1$. Further, $h(u) \in\left(h \jmath(y), h_{\jmath}(x)\right)=(\varepsilon, \beta)$. On the other hand, $u \in\left(\imath_{\varepsilon}(z), \phi_{1} \jmath(z)\right)$ for some $z \in B_{\varepsilon}$ with $\|z\|=1$. Since $h \jmath(z)=\varepsilon$ and $h \phi_{1} \jmath(z)=\beta$, necessarily $u \in\left(\jmath(z), \phi_{1} \jmath(z)\right)$. Finally, $\|\jmath(z)\|=1,\left\|\imath_{\varepsilon}(z)\right\| \leq 1$ by Claim 1 and $\left\|\phi_{1} \jmath(z)\right\| \geq 1$ by the claim between the Claims 1 and 2. Thus $\|u\| \geq 1$, a contradiction.

Now, from the containment in Claim 2 we immediately obtain

$$
\imath_{\varepsilon}\left(B(0,1) \cap g^{-1}(\beta)\right) \subset \phi_{\beta} J\left(B(0,1) \cap g^{-1}(\varepsilon)\right)-\frac{\beta}{\varepsilon} z_{\varepsilon} \subset B(0,1)
$$

since $\imath_{\varepsilon}\left(\jmath(x)+\frac{\beta-\varepsilon}{\varepsilon} z_{\varepsilon}\right)=\imath_{\varepsilon} \jmath(x)=\jmath(x)-z_{\varepsilon}$ (for the first inclusion); and for every $x \in B(0,1) \cap g^{-1}(\varepsilon)$ one has $\phi_{\beta} \jmath(x)-\frac{\beta}{\varepsilon} z_{\varepsilon}=\jmath(x)+\frac{\beta-\varepsilon}{\varepsilon} z_{\varepsilon}-\frac{\beta}{\varepsilon} z_{\varepsilon}=\jmath(x)-z_{\varepsilon} \in B(0,1)$ (for the second). Therefore, if $\|x\| \leq 1$ and $g(x)=\beta>\varepsilon$, namely, if $x \in B(0,1) \cap g^{-1}(\beta)$ then we have just shown $\left\|i_{\varepsilon}(x)\right\| \leq 1$. This, in combination with Claim 1 proves

Claim 3. If $\|x\|=1$ and $g(x) \geq \varepsilon$ then $\left\|\imath_{\varepsilon}(x)\right\| \leq 1$.

We are ready to conclude that $\left\|\imath_{\varepsilon}\right\| \leq 1+2 \varepsilon$ can be obtained, and the only remaining case is when $\|x\|=1$ and $g(x)<\varepsilon$. An immediate consequence of the preceding arguments is that $\left\|z_{\varepsilon}\right\| \leq 2 \varepsilon / \beta<2$ : pick $\|v\|=1$ with $\varepsilon<g(v)=\beta<1$. Since $\left\|\imath_{\varepsilon}(v)\right\|=\left\|\jmath(v)-\frac{\beta}{\varepsilon} z_{\varepsilon}\right\| \leq 1$ it follows that $\left\|z_{\varepsilon}\right\| \leq 2 \frac{\varepsilon}{\beta}<2$. And from that we obtain $\left\|\phi_{\beta} z_{\varepsilon}\right\|=\left\|\frac{\beta}{\varepsilon} z_{\varepsilon}\right\|<2$. The proof concludes now: since

$$
\imath_{\varepsilon}(x)=\jmath(x)-\frac{g(x)}{\varepsilon} z_{\varepsilon}=\jmath(x)-\frac{g(x)}{\beta} \phi_{\beta} z_{\varepsilon}
$$

it follows that

$$
\left\|\imath_{\varepsilon}(x)\right\| \leq 1+\frac{2}{\beta}|g(x)| \leq 1+\frac{2 \varepsilon}{\beta} \leq 1+3 \varepsilon .
$$

just picking $\beta>2 / 3$.

We have proved that there exist, for every $\varepsilon>0,(1+3 \varepsilon)$-extensions to onecodimensional superspaces. We need to show now that there exist equal norm extensions to one-codimensional superspaces. The point is the equivalence between extension properties and properties of intersections of balls. Nachbin [29] (see also [33, Theorem 2.1]) proved that a Banach space $X$ is 1-injective if and only if every family of mutually intersecting balls has nonempty intersection. It can be attributed to Lindenstrauss that a Banach space is 1-separably injective if and only if every countable family of mutually intersecting balls has nonempty intersection: see the 
comments in [28, Proof of the theorem]. As well as it can be attributed to him, in general (see [7] and [8,5.2 and 5.5.4]), that a Banach space is (1, $)$-injective if and only if every family of size $<\aleph$ of mutually intersecting balls has nonempty intersection. A combination of those ideas with the arguments of Lindenstrauss in [27, Theorem 6.10] yields

LEMMA 2. If every sequence $B\left(x_{n}, r_{n}\right)$ of mutually intersecting balls is such that $\bigcap_{n} B\left(x_{n}, r_{n}+\varepsilon\right) \neq \emptyset$ for every $\varepsilon>0$ then any countable family $B\left(x_{n}, r_{n}\right)$ of mutually intersecting balls has $\bigcap_{n} B\left(x_{n}, r_{n}\right) \neq \emptyset$. The result remains valid for families of size $<\aleph$.

Let us remark that also Davis in [15, p.316] formulates the compactness argument for radius 1 balls in the forms: If every sequence $B\left(x_{n}, 1\right)$ of mutually intersecting balls is such that $\bigcap_{n} B\left(x_{n}, 1+\varepsilon\right) \neq \emptyset$ for every $\varepsilon>0$ then also every sequence $B\left(x_{n}, 1\right)$ of mutually intersecting balls is such that $\bigcap_{n} B\left(x_{n}, 1\right) \neq \emptyset$.

This means that a Banach space $X$ is 1-separably injective if and only if, for every $\varepsilon>0$, every separable subspace $S \subset \ell_{\infty}$ and every $x \in \ell_{\infty}$, every norm one operator $\tau: S \rightarrow X$ admits an extension $\tau_{\varepsilon}: S+[x] \rightarrow X$ with $\left\|\tau_{\varepsilon}\right\| \leq 1+\varepsilon$. The $(1, \aleph)$-injective) version is easy to reformulate too. This concludes the proof.

The geometrical conclusion of the previous proof is:

COROllary 1. Let $\aleph$ be a cardinal and let $X$ be a Banach space. Every family of size $<\aleph$ of mutually intersecting balls has nonempty intersection if and only if every family of size $<\aleph$ of mutually intersecting balls of radius 1 has nonempty intersection.

Proof. One implication is obvious. As for the other, let us again simplify the exposition working with $\aleph=\aleph_{1}$. If every countable family of mutually intersecting balls of radius 1 has nonempty intersection then $J_{s}(X)=1$ : indeed, let $A$ be a separable set with diameter 2 and let $\left\{x_{n}\right\}_{n}$ be a countable dense subset of $A$. The intersection of each two balls $B\left(x_{i}, 1\right) \cap B\left(x_{j}, 1\right)$ is nonempty and thus there is some $p \in \cap_{n} B\left(x_{n}, 1\right)$. This means that $A \subset B(p, 1+\varepsilon)$ and the radius of $A$ is at most $1+\varepsilon$, which implies $J_{s}(X)=1$. Thus $X$ is 1 -separably injective by Theorem 1 , hence every countable family of mutually intersecting balls has nonempty intersection.

Thus, the difference between 1-separable (resp. $(1, \aleph)$ ) injectivity and having separable Jung (resp. $J_{\aleph}$ ) constant 1 is whether intersection properties of sequences (resp. families of size $<\aleph$ ) of radius 1 balls pass to arbitrary sequences (resp. families of size $<\aleph$ ) of balls, as it is the case.

\section{Uses and Applications}

2.1. Bounded sets without center. Thus, in 1-separably injective spaces that are not 1-injective there are closed bounded sets with diameter 2 but radius 
$>1$ and these cannot be separable. The three main examples in the literature (see [8]) of 1-separably injective spaces that are not 1-injective are:

(1) Given an uncountable set $\Gamma$ then the space $\ell_{\infty}^{c}(\Gamma)$ of bounded functions with countable support.

(2) The space $\ell_{\infty} / c_{0}$.

(3) Ultrapowers of Lindenstrauss spaces with respect to countably incomplete ultrafilters on $\mathbb{N}$.

It is worth to carefully exam why such exotic bounded sets exist in those spaces.

- Fix as $\Gamma$ a set of size the continuum c. The set $V$ of elements of $\ell_{\infty}^{c}(\Gamma)$ taking values in $[0,1]$ has diameter 1 but no center for radius $1 / 2$ balls since such center should be the constant function, which is not in $\ell_{\infty}^{c}(\Gamma)$. On the other hand splitting $\Gamma=\Gamma_{1} \cup \Gamma_{2}$ with both $\Gamma_{i}$ uncountable then the set $A_{1} \cup-A_{2}$ with $A_{i}=\left\{f: \operatorname{supp} f \subset \Gamma_{i}\right\}$ has diameter 1 and radius 1 (this example is taken from [2]). Hence $J\left(\ell_{\infty}^{c}(\Gamma)\right)=2$.

- An example of a diameter 1 set with radius 1 in $\ell_{\infty} / c_{0}$ has been kindly provided to us by Manuel González: Suppose that $\left\{r_{i}: i \in I\right\}$ is the set of all branches in the dyadic tree $\Delta$, so that $|I|=\mathfrak{c}$. We enumerate the nodes of $\Delta$ in the usual way, so that we can assume $\Delta=\mathbb{N}$. Let $1_{i}$ be the characteristic function of $r_{i}$, so that we can assume $1_{i} \in \ell_{\infty}$. As it is well-known, the images $g_{i}$ of $1_{i}$ in $\ell_{\infty} / c_{0}$ generate a subspace isometric to $c_{0}(I)$ because the intersection of two different branches is finite. As a consequence, for every $\varepsilon \in\{-1,1\}^{I}$ the set $A_{\varepsilon}=\left\{\varepsilon(i) \cdot g_{i}: i \in I\right\}$ has diameter 1 . Now, for each $r<1$ there is a choice of signs $\varepsilon \in\{-1,1\}^{I}$ so that $A_{\varepsilon}$ cannot be contained in a ball $B\left(h_{\varepsilon}, r\right)$ since otherwise

$$
\begin{aligned}
2 & =\left\|\varepsilon(i) \cdot g_{i}-\eta(i) \cdot g_{i}\right\| \\
& \leq\left\|\varepsilon_{i} \cdot g_{i}-h_{\varepsilon}\right\|+\left\|h_{\varepsilon}-h_{\eta}\right\|+\left\|h_{\eta}-\eta_{i} \cdot g_{i}\right\| \\
& \leq 2 r+\left\|h_{\varepsilon}-h_{\eta}\right\|
\end{aligned}
$$

yields $\left\|h_{\varepsilon}-h_{\eta}\right\| \geq 2(1-r)$. Thus, $\left\{h_{\varepsilon}: \varepsilon \in\{-1,1\}^{I}\right\}$ is a $2(1-r)$-separated family of cardinality $2^{\mathfrak{c}}$ in a space, $\ell_{\infty} / c_{0}$, of density character $\mathfrak{c}$, which is impossible. Consequently $J\left(\ell_{\infty} / c_{0}\right)=2$.

- The case of ultrapowers is quite similar to the previous one.

2.2. Separable injectivity and the Grünbaum expansion constant. Grünbaum considers in [21, 22] the expansion constant $E(X)$ of a Banach space $X$ as the infimum of those $\lambda>0$ such that whenever one has a family $B\left(X_{i}, r_{i}\right)$ of mutually intersecting balls then $\cap_{i} B\left(x_{i}, \lambda r_{i}\right) \neq \emptyset$. While [21] is devoted to the study of the expansion constant in finite-dimensional spaces, [22 considers the expansion constant in infinite dimensional spaces and establishes that the extension constant 
coincides with the projection constant defined as

$$
p(X)=\sup _{Y} \inf _{P}\{\|P\|: P: Y \rightarrow X\}
$$

where $Y$ runs over all 1-codimensional superspaces of $X$ and $P$ over all projections of $Y$ onto $X$. It is therefore clear that $E(X)=1$ and the infimum is attained (what Grünbaum calls " $E(X)=1$ is exact") if and only if $X$ is 1-injective. Since one has [21, (iii)] that $J(X) \leq E(X)$ it turns out that $E(X)=1$ implies $J(X)=1$ so $X$ is 1-injective and thus $E(X)=1$ is exact. This is relevant since Grünbaum provides in [22] an example of a space $X$ for which $E(X)=2=J(X)$ and such that $J(X)$ is exact but $E(X)$ is not.

If one defines the separable extension constant $E_{s}(X)$ (and its corresponding cardinal versions $\left.E_{\aleph}(X)\right)$ by simply restricting the size of the family of balls, one still has $J_{s}(X) \leq E_{s}(X)$ (and, in general, $E_{\aleph}(X) \leq J_{\aleph}(X)$ ) and therefore, after Theorem 1 one gets: $\quad X$ is 1-separably injective if and only if $E_{s}(X)=1$ is exact, which is [8, Lemma 2.30]. In general, when $X$ is $\lambda$-separably injective then $E_{s}(X) \leq \lambda$ [8, Lemma 2.33]. It is also clear that there is no simple version of the projection constant $p$ that characterizes $J_{s}$.

2.3. On the stability of the Jung constants. Most of the known results about stability of the Jung constant can be found in [2] and most of them treat the case of reflexive spaces and thus are not interesting for us. It was already shown by Amir [2, Prop.1.1] that every finite-codimensional subspace of a $C(K)$ space with $K$ not extremally disconnected (i.e., every non 1-injective $C(K)$-space) has Jung constant 2, which proves Franchetti's conjecture [18 that $J(C(K))<$ 2 implies $J(C(K))=1$. A version of Amir's theorem which somehow extends Franchetti's conjecture to $J_{s}$ appears in [8, Proposition 2.4]. Recall from [8] that a $C(K)$ space is 1-separably injective if and only if $K$ is an $F$-space (cf. [8] for its definition). Amir proves that $E_{s}(C(K))<2$ implies that $K$ is an $F$-space, therefore $C(K)$ is 1-separably injective, and thus $J_{s}(C(K))=1$. Cardinal modifications of [8, Proposition 2.4] obtained taking [8, Proposition 5.12] into account yield that $E_{\aleph}(C(K))<2$ implies that $K$ is an $F_{\aleph}$-space [8, Definition 5.19], and therefore $C(K)$ is $(1, \aleph)$-separably injective [8, Theorem 5.16], and thus $J_{\aleph}(C(K))=1$. This suggests the question of whether $J_{\aleph}(\mathcal{C})<2$ implies $J_{\aleph}(\mathcal{C})=1$ for a space $C(K)$ of continuous functions on a compact space $K$.

Given a Banach space parameter, say $\eta(\cdot)$, it is usually a relevant question whether $\eta(X)=\eta\left(X^{* *}\right)$. For instance, the problem of whether $T(X)=T\left(X^{* *}\right)$ for the thickness or Whitley constant [32] was posed in [13] and negatively solved in [1], while the same problem for the Kottman constant was posed in [13] and negatively solved in [11]. The situation for Jung constants is not different, although in this case we already know that $J(X)$ (resp. $\left.J_{s}(X)\right)$ and $J\left(X^{* *}\right)\left(\operatorname{resp} . J_{s}\left(X^{* *}\right)\right)$ can be different. Indeed, $J\left(c_{0}\right)=2=J_{s}\left(c_{0}\right)$ while $J\left(\ell_{\infty}\right)=J_{s}\left(\ell_{\infty}\right)=1$. In general, given a countably incomplete ultrafilter $\mathcal{U}$ on $\mathbb{N}$, one has $J_{s}(C[0,1])=2$ but 
$J_{s}\left(C[0,1]_{u}\right)=1$ since according to $[\mathbf{7}, \mathbf{8}]$ the ultrapower of a Lindenstrauss space is 1-separably injective, as we have already mentioned. On the other hand, $J\left(\ell_{\infty}\right)=1$ but $J\left(\left(\ell_{\infty}\right) u\right)=2$ since, again according to [7, 8] no infinite-dimensional ultrapower is injective.

2.4. 1-separably injective spaces. In [8, Problem 7] it is posed the problem of the existence of 1-separably injective subspaces of $\ell_{\infty}$ not isomorphic to $\ell_{\infty}$. We can present a partial answer:

Lemma 3. A 1-separably injective subspace $\Theta \subset \ell_{\infty}$ containing the canonical copy of $c_{0}$ is $\ell_{\infty}$.

Proof. Every point $x=\left(x_{n}\right) \in \ell_{\infty}$ is the unique center of a countable set of $c_{0}$ : the set $\left\{\left(x_{n}+\|x\|\right) e_{n},\left(x_{n}-\|x\|\right) e_{n}: n \in \mathbb{N}\right\}$ has diameter $2\|x\|$ and radius $\|x\|$ with $x$ the only center.

The result can be extended to subspaces $\Theta$ whose unit ball is weak ${ }^{*}$-dense in the unit ball of $\ell_{\infty}$, but one is still far from showing even that no 1-separably injective subspace of $\ell_{\infty}$ containing an isometric copy of $c_{0}$, different from $\ell_{\infty}$, exists. The following application was suggested by F. Cabello:

Corollary 1. $\beta \mathbb{N}$ is the only compactification of $\mathbb{N}$ that is an F-space

Proof. Let $\gamma \mathbb{N}$ be a compactification of $\mathbb{N}$. An extension of the canonical map $\mathbb{N} \rightarrow \gamma \mathbb{N}$ yields a quotient map $\beta \mathbb{N} \rightarrow \gamma \mathbb{N}$. Thus $C(\gamma \mathbb{N})$ is a subspace of $C(\beta \mathbb{N})=\ell_{\infty}$ that contains the canonical copy of $c_{0}$. If $\gamma \mathbb{N}$ is an $F$-space then $C(\gamma \mathbb{N})$ is 1-separably injective, and thus it must be isomorphic to $\ell_{\infty}$.

Let now $X$ be a subspace of $\ell_{\infty}$. Consider the set $\mathcal{C}_{0} \subset X^{\mathbb{N}}$ of all countable families of elements of $X$ such that $\left\|x_{n}-x_{m}\right\| \leq 2$ for $n \neq m$; any family $F \in \mathcal{C}_{0}$ has (at least) a center $z(F) \subset \ell_{\infty}$. Let $z(X)$ be a set containing a center for each family in $\mathcal{C}_{0}$. Set $X_{1}=[X+z(X)] \subset \ell_{\infty}$ and proceed inductively. Given a countable ordinal $\alpha$ for which $X_{\alpha} \subset \ell_{\infty}$ has been chosen, if $\alpha$ is not a limit ordinal then let $\mathcal{C}_{\alpha}$ be the set of all countable families of elements of $X_{\alpha}$ such that $\left\|x_{n}-x_{m}\right\| \leq 2$ for $n \neq m$. For each $F \in \mathcal{C}_{\alpha}$ let $z\left(X_{\alpha}\right)$ be a set of centers of the families in $\mathcal{C}_{\alpha}$. Finally set $X_{\alpha+1}=\left[X_{\alpha}+z\left(X_{\alpha}\right)\right]$ in $\ell_{\infty}$. If $\alpha$ is a limit ordinal work with the closure of $\bigcup_{\beta<\alpha} X_{\beta}$. Iterate the process $\omega_{1}$ steps to get a 1-separably injective subspace $X_{\omega_{1}}$ of $\ell_{\infty}$ : indeed, giving a mutually intersecting sequence of balls $\left\{B\left(x_{n}, 1\right): n \in \mathbb{N}\right\}$ the set $\left\{x_{n}: n \in \mathbb{N}\right\}$ is contained in some $X_{\alpha}$, hence it has a center in $X_{\alpha+1}$ and thus in $X_{\omega_{1}}$. Thus, $J_{s}\left(X_{\omega_{1}}\right)=1$ and, by Theorem 1, $X_{\omega_{1}}$ is 1-separably injective. There are however many possible choices for the successive sets of centers, so the final resulting space $X_{\omega_{1}}$ is, in principle, not unique. In fact, if [8, Problem 7] above has a negative solution and no 1-separably injective subspaces of $\ell_{\infty}$ different from $\ell_{\infty}$ exist, all spaces $X_{\omega_{1}}$ must be the isomorphic to $\ell_{\infty}$. Let us now exam what occurs when the centers are chosen outside $\ell_{\infty}$. Recall that given a Banach space $X$ 
there are several constructions in the literature $[\mathbf{5}, \mathbf{6}, \mathbf{8}, \mathbf{1 4}$ of 1-separably injective spaces $\mathcal{S}(X)$ containing an isometric copy of $X$ and with the additional property that every operator $\tau: X \rightarrow \mathcal{S}$ from $X$ into a 1-separably injective space $\mathcal{S}$ admits an equal norm extension $\mathcal{S}(X) \rightarrow \mathcal{S}$. These were called 1 -separably injective covers of $X$. One has:

LEMMA 4.

(1) Under $\mathrm{CH}$, there is a subspace of $G \subset \ell_{\infty}$ such that no 1-separably injective cover $\mathcal{S}(G)$ can be isomorphic to $\ell_{\infty}$ or a subspace of $\ell_{\infty}$.

(2) Under MA $+\mathfrak{c}=\aleph_{2}$, any 1-separably injective cover $\mathcal{S}\left(c_{0}\right)$ of $c_{0}$ is not isomorphic to $\ell_{\infty}$ and is not a subspace of $\ell_{\infty}$.

Proof. We prove first assertion (1). Consider any embedding $\mathcal{G} \rightarrow C[0,1]$ where $\mathcal{G}$ denotes Gurariy space and form the resulting exact sequence

$$
0 \longrightarrow \mathcal{G} \longrightarrow C[0,1] \longrightarrow Q \longrightarrow 0
$$

No ultrapower of this sequence splits since, by the results in [4, 8], no ultrapower of the Gurariy space is a complemented subspace of any $C(K)$-space. Write $Q u$ as a quotient of $\ell_{1}(\mathfrak{c})$ with kernel $K$ and form the commutative diagram

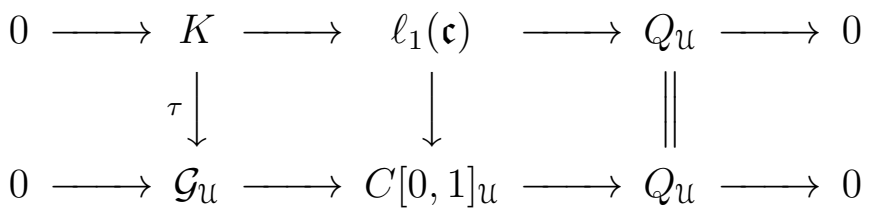

in which the operator $\tau$ cannot be extended to an operator $\ell_{1}(\mathfrak{c}) \rightarrow \mathcal{G}_{\mathfrak{u}}$. Since $\ell_{1}(\mathfrak{c})$ is a subspace of $\ell_{\infty}, \tau$ cannot be extended to $\ell_{\infty}$. Now, observe that if a subspace $X$ of $\ell_{\infty}$ admits an embedding $i: X \rightarrow \ell_{\infty}$ such that operators from $X$ into a space $E$ can be extended to $\ell_{\infty}$ through $i$ then every embedding $j: X \rightarrow \ell_{\infty}$ enjoys the same property as $i$. Thus, a subspace $X$ of $\ell_{\infty}$ such that some $\mathcal{S}(X)$ is isomorphic to $\ell_{\infty}$ admits an embedding $X \rightarrow \ell_{\infty}$ with the property that every operator $X \rightarrow E$ from $X$ into any 1 -separably injective space can be extended to $\ell_{\infty}$. And consequently, the same occurs to any embedding $X \rightarrow \ell_{\infty}$. In conclusion, pick $X=K$ to get that $\mathcal{S}(K)$ cannot be isomorphic to $\ell_{\infty}$.

Pick now $G=c_{0}+K$. Any 1-separably injective subspace of $\ell_{\infty}$ containing $G$ must therefore be $\ell_{\infty}$ by Lemma 3. On the other hand, since $K$ is Schur, $c_{0}$ and $K$ are incomparable and thus $c_{0}+K=c_{0} \oplus K$. There is therefore an operator $c_{0}+K \rightarrow \mathcal{G}_{u}$ that cannot be extended to $\ell_{\infty}$ and thus no 1-separably injective cover of $G$ can be a subspace of $\ell_{\infty}$.

To prove (2) we need to consider the 1-separably injective space $\mathcal{A K}$ that does not contain $\ell_{\infty}$ constructed by Avilés and Koszmider [9] under the axioms MA + $\mathfrak{c}=\aleph_{2}$. Since $\mathcal{A K}$ is separably injective, it contains $c_{0}[\mathbf{5}$, 8, but the inclusion $c_{0} \rightarrow \mathcal{A K}$ cannot be extended to an operator $\ell_{\infty} \rightarrow \mathcal{A K}$ : indeed, any such extension should be, by Rosenthal's theorem [30], either a weakly compact operator, which is 
impossible, or an isomorphism on a copy of $\ell_{\infty}$, which is impossible too since $\mathcal{A K}$ does not contain $\ell_{\infty}$. Consequently, $\mathcal{S}\left(c_{0}\right) \neq \ell_{\infty}$. By Lemma $3, \mathcal{S}\left(c_{0}\right)$ cannot be a subspace of $\ell_{\infty}$.

The difference between the two results is that the subspace $G$ of (1) cannot be separable since, under $\mathrm{CH}, \ell_{\infty}$ is a 1-separable injective cover of every separable space. A related topic is the open problem of whether 1-separably injective spaces with density character at most $\mathfrak{c}$ must be quotients of $\ell_{\infty}$. Observe that under $\mathrm{MA}+\mathfrak{c}=\aleph_{2}$ the space $\mathcal{A K}$ cannot be a quotient of $\ell_{\infty}$.

\section{The interplay between the Jung and Kottman constants: extension of Lipschitz maps}

If $X$ is an infinite-dimensional Banach space with unit ball $B(X)$, the Kottman constant [26] of $X$ is defined as

$$
K(X)=\sup _{\left(x_{n}\right) \in B(X)} \operatorname{sep}\left(x_{n}\right)
$$

where, for a given sequence $\left(x_{n}\right)$, we define $\operatorname{sep}\left(\left(x_{n}\right)_{n}\right)=\inf _{n \neq m}\left\|x_{n}-x_{m}\right\|$. A well known result of Elton and Odell [17] asserts that $K(X)>1$ for every infinitedimensional Banach space $X$. The finite Kottman constant is defined as

$$
K_{f}(X)=\sup \{r>0: \forall n \in \mathbb{N} \quad \exists A:|A|=n \text { and } r \text { - separated }\}
$$

We define the Lipschitz expansion constants: $e(X, Z)$ (resp. $\left.e_{1}(X, Z)\right)$ is the infimum of all $\lambda>0$ such that for every subset $M$ of $X$ (resp. every subset $M$ and every point $x \in X$ ) every Lipschitz map $f: M \rightarrow Z$ admits a Lipschitz extension $F: X \rightarrow Z$ (resp. $F: M \cup\{x\} \rightarrow Z$ ) with $\operatorname{Lip}(F) \leq \lambda \operatorname{Lip}(f)$. It will be useful for us to define the constants $e_{1}^{s}(X, Z)$ (resp. $\left.e_{1}^{f}(X, Z)\right)$ as the versions of $e_{1}(X, Z)$ obtained allowing only separable (resp. finite) $M$. It is clear that $e_{1}^{f} \leq e_{1}^{s} \leq e_{1}$. One has

Proposition 1. For every couple $Y, X$ of infinite dimensional Banach spaces one has

(1) $K(Y) J_{s}(X) \leq 2 e_{1}^{s}(Y, X)$.

(2) $K_{f}(Y) J_{f}(X) \leq 2 e_{1}^{f}(Y, X)$.

(3) $K_{f}(Y) J_{s}(X) \leq 2 e_{1}^{s}\left(Y, X^{* *}\right)$.

The inequalities above are sharp and $J_{s}$ cannot be replaced by $J$; precisely, $K(Y) J(X) \leq 2 e_{1}(Y, X)$ does not hold.

Proof. Let $\varepsilon>0$. Consider in $X$ a bounded countable set $A$ such that $\frac{2 r(A)}{\delta(A)}>$ $J_{s}(X)-\varepsilon$ and $\delta(A)=K(X)-\varepsilon$. Choose in the unit ball of $Y$ an infinite, countable set $C=\left\{y_{1}, \ldots, y_{n}, \ldots\right\}$ such that $K(Y)-\varepsilon \leq\left\|y_{i}-y_{j}\right\| \leq K(Y)+\varepsilon$ for every pair 
$i, j \in N ; i \neq j$ (see [16, 3.4 Lemma]). Consider any bijective map $\tau: C \rightarrow A$. This map is 1-Lipschitz since

$$
\left\|\tau y_{i}-\tau y_{j}\right\| \leq \delta(A) \leq\left\|y_{i}-y_{j}\right\|
$$

Let $T: C \cup\{0\} \rightarrow X$ be a Lipschitz extension of $\tau$ with Lipschitz constant not greater than $e_{1}^{s}(Y, X)+\varepsilon$. It turns out that

$$
\begin{aligned}
\frac{1}{2}\left(J_{s}(X)-\varepsilon\right)(K(Y)-\varepsilon) & =\frac{1}{2}\left(J_{s}(X)-\varepsilon\right) \delta(A) \\
& <r(A) \\
& \leq \sup \left\{\left\|T(0)-\tau y_{i}\right\|: i \in N\right\} \leq e_{1}^{s}(Y, X)+\varepsilon
\end{aligned}
$$

Letting $\varepsilon \rightarrow 0$ proves part (1) of the lemma.

To obtain the finite version (2) we need a result which is likely to be known, but for which we could only find an abstract formulation in [23, Cor. 1.4], so we include a simple proof here for the sake of completeness.

Lemma 5. Let $X$ be a Banach space and let $\varepsilon>0$. For every $n \in \mathbb{N}$ it is possible to choose in the unit ball of $X$ a set $\left\{x_{1}, \ldots, x_{n}\right\}$ such that $K_{f}(X)-\varepsilon \leq\left\|x_{i}-x_{j}\right\| \leq$ $K_{f}(X)+\varepsilon$ for every pair $i, j=1, \ldots, n ; i \neq j$.

Proof. For some $n_{1} \in \mathbb{N}$ one cannot find $n_{1}$ points in $B(X)$ which are $\left(K_{f}(X)+\right.$ $\varepsilon)$-separated. According to the finite Ramsey theorem, there is a number $R\left(n, n_{1}\right)$ such that whenever one has $n_{2} \geq R\left(n_{1}\right)$ points which are $\left(K_{f}(X)-\varepsilon\right)$-separated, there must be either $n$ points whose mutual distance is between $K_{f}(X)-\varepsilon$ and $K_{f}(X)+\varepsilon$, or $n_{1}$ points $K_{f}(X)+\varepsilon$-separated, which is impossible. So only the first case holds, which proves the assertion.

The rest of the argument is as before.

Regarding the proof of (3), it was observed in [12] that $K_{f}(Y)=K\left(Y_{u}\right)$ for every countably incomplete ultrafilter $\mathcal{U}$. Therefore, inequality (1) becomes

One has:

$$
\frac{1}{2} K_{f}(Y) J_{s}(X) \leq e_{1}^{s}\left(Y_{\mathfrak{u}}, X\right)
$$

Claim. $e_{1}^{s}\left(Y_{u}, X\right) \leq e_{1}^{s}\left(Y, X^{* *}\right)$.

Proof of the Claim. Let $M \subset Y_{u}$ be a countable subset, $x \notin M$ and $f: M \rightarrow X$ a Lipschitz map. Assume that $M=\left\{\left[m_{n}^{k}\right]: k \in \mathbb{N}\right\}$ and $x=\left[x_{n}\right]$. Form the countable subsets $M_{k}=\left\{m_{n}^{k}: n \in \mathbb{N}\right\} \subset Y$ and consider the restriction $f_{k}$ of $f$ to the set $M_{k}$ inside the natural (diagonal) copy of $Y$ inside $Y_{u}$. Let $F_{k}$ be an extension to $M_{k} \cup\left\{x_{k}\right\}$ with Lipschitz constant $e_{1}^{s}(Y, X)+1 / k$. Form the element $\left[F_{k}\right]: M \cup\{x\} \rightarrow X_{\mathcal{u}}$ given by $\left[F_{k}\right]\left[z_{n}\right]=\left[F_{k}\left(z_{k}\right)\right]$, which is a Lipschitz map with Lipschitz constant $e_{1}^{s}(Y, X)$. Pick a norm one projection $X_{\mathfrak{U}} \rightarrow X^{* *}$ to get a Lipschitz map $P\left[F_{k}\right]: M \cup\{x\} \rightarrow X^{* *}$ with Lipschitz constant $e_{1}^{s}(Y, X)$. 
One thus gets $\frac{1}{2} K_{f}(Y) J_{s}(X) \leq e_{1}^{s}\left(Y, X^{* *}\right)$.

None of the second group of inequalities can hold: pick as $X$ any 1-separably injective space that is not 1-injective and $Y$ separable such that $K(Y)=2$. The inequality $K(Y) J(X) \leq e_{1}^{s}(Y, X)$ forces $J(X)=1$ which cannot be. The inequality $K(Y) J(X) \leq e_{1}(Y, X)$ also fails: pick $Y=c_{0}$ and $X=\ell_{\infty} / c_{0}$; then $K\left(c_{0}\right)=$ $J\left(\ell_{\infty} / c_{0}\right)=2$ and $e_{1}\left(c_{0}, \ell_{\infty} / c_{0}\right)=e_{1}^{s}\left(c_{0}, \ell_{\infty} / c_{0}\right)=1$.

\section{Applications.}

4.1. Kalton proved in [25, Proposition 5.8] the unexpected result that

$$
K(X) \leq e\left(X, c_{0}\right)
$$

for every infinite dimensional Banach space $X$. See [31] for an extension to $\alpha$-Hölder maps. We derive now from Proposition 1 a simple proof for an improved version.

Proposition 2. For every infinite dimensional Banach space $X$ one has .

$$
K(X) \leq e_{1}^{s}\left(X, c_{0}\right)
$$

Proof. The result follows from $J_{s}\left(c_{0}\right)=2$ and $K(X) J_{s}\left(c_{0}\right) \leq 2 e_{1}^{s}\left(X, c_{0}\right)$.

4.2. Kalton shows in 25] that if $e_{1}(Y, X)=\lambda$ then norm one operators from subspaces of $Y$ to $X$ admit, for each $\varepsilon>0$, an extension to one more dimension with norm at most $\lambda+\varepsilon$. The argument is simple: pick $A$ a subspace of $Y$ and $b \notin A$. If a norm one operator $\tau: A \rightarrow X$ extends to a Lipschitz map $L: A \cup\{b\} \rightarrow X$ with Lipschitz constant $\lambda$, set $T: A+[b] \rightarrow X$ the operator $T(b)=L(b)$. Then for every $a \in A$ one has $\|T(a-b)\|=\|L a-L b\| \leq \lambda\|a-b\|$, from where $\|T\| \leq \lambda$. One thus has:

Lemma 6. A Banach space $X$ such that $e_{1}(Y, X)=1$ for every Banach space $Y$ must be 1-injective.

Let us show how this result can be completed. Recall that the argumentation above cannot be used for 1-separable injectivity. One has:

THEOREM 2.

- A Banach space $X$ is 1-injective if and only if $e_{1}(Y, X)=1$ for all Banach spaces $Y$.

- A Banach space $X$ is 1-separably injective if and only if $e_{1}^{s}(Y, X)=1$ for all Banach spaces $Y$.

Proof. To prove the first assertion we just need to obtain the converse of Kalton's lemma 6, and this is consequence of

LEMma 7. If all operators $E \rightarrow X$ extend with the same norm to operators $Y \rightarrow X$ whenever $\operatorname{dim} Y / E=1$ then Lipschitz maps $M \rightarrow X$ extend to Lipschitz maps $M \cup\{p\} \rightarrow X$ with the same Lipschitz constant. 
Proof. Let $\operatorname{Lip}(M, \mathbb{R})_{0}$ be the space of Lipschitz maps $\ell: M \rightarrow \mathbb{R}$ such that $\ell\left(m_{0}\right)=0$ for a fixed $m_{0} \in M$. It is a Banach space endowed with the natural norm $\|\ell\|=\operatorname{Lip}(\ell)$ where Lip denotes the Lipschitz constant of $\ell$. Let $\Delta: M \rightarrow$ $\operatorname{Lip}(M, \mathbb{R})_{0}^{*}$ be the canonical Lipschitz map given by $\Delta_{m}(\ell)=\ell(m)$. The Lipschitzfree space [20] $\mathcal{F}(M)$ is the closure of the image $\delta(M)$. It has the property that for every Lipschitz map $L: M \rightarrow X$ such that $L\left(m_{0}\right)=0$ there is an operator $\phi_{L}: \mathcal{F}(M) \rightarrow X$ such that $\phi_{L} \Delta=L$ and $\left\|\phi_{L}\right\|=\operatorname{Lip}(L)$.

Now, let $L: M \rightarrow X$ be a Lipschitz map. With a translation there is no loss of generality assuming that $L\left(m_{0}\right)=0$. Since $\mathcal{F}(M)$ is a one-codimensional subspace of $\mathcal{F}(M \cup p)$, the operator $\phi_{L}: \mathcal{F}(M) \rightarrow X$ extends with the same norm to an operator $\widehat{\phi_{L}}: \mathcal{F}(M \cup p) \rightarrow X$, and thus $\widehat{\phi_{L}} \Delta$ is a Lipschitz map extending $L$ with the same Lipschitz constant.

To prove the second equivalence we use the inequality $K(Y) J_{s}(X) \leq e_{1}^{s}(Y, X)$ to get that a Banach space $X$ such that $e_{1}^{s}(Y, X)=1$ for some Banach space $Y$ with $K(Y)=2$ must have $J_{s}(X)=1$ and thus must be 1-separably injective. Conversely, if a Banach space is 1-separably injective then $e_{1}(Y, X)=1$ for every separable Banach space $Y$. Now, if $e_{1}(Y, X)=1$ for all separable Banach spaces $Y$ then $e_{1}^{s}(Y, X)=1$ for all Banach spaces $Y$.

\section{References}

[1] T. A. Abrahamsen, J. Langemets, V. Lima, O. Nygaard, On thickness and thinness of Banach spaces, Houston J. Math. 41 (2015) 97 - 111. 357

[2] D. Amir, On Jung's constant and related constants in normed linear spaces, Pacific J. Math. 118 (1985) 1-16.

[3] J. Appell, C. Franchetti, E.M. Semenov, Estimates for the Jung constant in Banach lattices, Israel J. Math. 116 (2000) 171-187.

[4] A. Avilés, F. Cabello, J.M.F. Castillo, M. González, Y. Moreno, Corrigendum to "On separably injective Banach spaces, Adv. Math. 234 (2013) 192-216", Adv. Math. 318 (2017) 737-747.

[5] A. Avilés, F. Cabello, J.M.F. Castillo, M. González, Y. Moreno, On separably injective Banach spaces, Adv. Math. 234 (2013) 192-216.

[6] A. Avilés, F. Cabello, J.M.F. Castillo, M. González, Y. Moreno, On Banach spaces of universal disposition, J. Funct. Anal. 261 (2011) 2347-2361.

[7] A. Avilés, F. Cabello, J.M.F. Castillo, M. González, Y. Moreno, ※-injective Banach spaces and $\aleph$-projective compacta, Rev. Mat. Ibeoramericana 31 (2015) 575-600.

[8] A. Avilés, F. Cabello, J.M.F. Castillo, M. González, Y. Moreno, Separable injective Banach spaces, Lecture Notes in Mathematics 2132 (2016) Springer-Verlag.

[9] A. Avilés, P. Koszmider, A 1-separably injective space that does not contain $\ell_{\infty}$, Bull. London Math. Soc. 50 (2018) 249-260.

[10] José M. Bayod, M. Concepción Masa, Coeficientes de Chebyshev en espacios de funciones continuas, Proceedings of the XIVth Spanish-Portuguese Conference in Mathematics, vols I-II, pp. 233-235; Puerto de la Cruz 1989, Univ. La Laguna 1990. MR 1112877.

[11] J.M.F. Castillo, M. González, P.L. Papini, New results on Kottman's constant, Banach J. Math. Anal. 11 (2017) 348-362. 
[12] J.M.F. Castillo, P. L. Papini, On Kottman's constants in Banach spaces, Banach Center Publications 92 (2011) 75-84.

[13] J.M.F. Castillo, P.L. Papini, M.A. Simões, Thick coverings for the unit ball of a Banach space, Houston J. Math. 41 (2015) 177-186.

[14] J.M.F. Castillo, J. Suárez, On $\mathcal{L}_{\infty}$-envelopes of Banach spaces, J. Math. Anal. Appl. 394 (2012) 152-158.

[15] W.J. Davis, A characterization of $\mathcal{P}_{1}$-spaces, J. Approx. Theory 21 (1977) 315-318.

[16] T. Domínguez Benavides, Some properties of the set and ball measures of non-compactness and applications, J. London Math. Soc. 34 (1986) 120-128.

[17] J. Elton, E. Odell, The unit ball of every infinite-dimensional normed linear space contains a $(1+\varepsilon)$-separated sequence, Colloq. Math. 44 (1981) 105-109.

[18] C. Franchetti, Relations between the Jung constant and a certain projection constant in Banach spaces, Ann. Univ. Ferrara, 23 (1977) 39-44.

[19] C. Franchetti, Projection onto hyperplanes in Banach spaces, J. Approx. Theory 38 (1983) 319-323.

[20] G. Godefroy, N. J. Kalton, Lipschitz-free Banach spaces, Studia Math. 150 (2003) 121-140.

[21] B. Grünbaum, On some covering and intersection properties in Minkowski spaces, Pacific J. Math. 9 (1959) 487-494.

[22] B. Grünbaum, Some applications of expansion constants, Pacific J. Math. 10 (1960) 193 - 201.

[23] W. van Heijst, Separated sequences in Banach spaces, Thesis, Delft, Nederland June 2011.

[24] H.W.E. Jung, Über die kleinste Kugel, die eine räumliche Figur einschliesst, J. Reine Angewandte. Math. 123 (1901) 241-257.

[25] N. J. Kalton, Extending Lipschitz maps into C(K)-spaces, Israel J. Math. 162 (2007) 275-315.

[26] C. A. Kottman, Packing and reflexivity in Banach spaces, Tran. Amer. Math. Soc. 150 (1970) $565-576$.

[27] J. Lindenstrauss, On the extension of compact operators, Mem. Amer. Math. Soc. 48 (1964).

[28] J. Lindenstrauss, On the extension of operators with range in a $C(K)$-space, Proc. Amer. Math. Soc. 15 (1964) 218-225.

[29] L. Nachbin, A theoem of Hahn-Banach type for linear transformations, Trans. Amer. Math. Soc. 68 (1950) 28-46.

[30] H.P. Rosenthal, On relatively disjoint families of measures, with some applications to Banach space theory, Studia Math. 37 (1970) 13-36.

[31] J. Suárez de la Fuente, The Kottman constant for $\alpha$-Hölder maps, Canad. Math. Bull. 60 (2017) 855-860.

[32] R. Whitley, The size of the unit sphere, Canad. J. Math. 20 (1968) $450-455$.

[33] M. Zippin, Extension of bounded linear operators, in Handbook of the Geometry of Banach spaces Vol 2, Edited by W.B. Johnson and J. Lindenstrauss, Elsevier, (2003), 1703-1741.

Instituto de Matemsstica de la Universidad de Extremadura, Avenida de Elvas, 06071-BADAJOZ, Spain

E-mail address: castillo@unex.es

via Martucci 19, 40136 Bologna, Italia

E-mail address: pierluigi.papini@unibo.it 